\title{
ON THE NORMALITY OF AN ANALYTIC OPERATOR ${ }^{1}$
}

\section{JOHN BUTLER}

1. Introduction. Let $T(z)$ be a function of the complex variable $z$, $|z|<\gamma$, whose function values are closed operators with fixed domain $\mathfrak{D}$ independent of $z$ on Hilbert space $\mathfrak{S}$, and such that for all $f \in \mathfrak{D}$, $T(z) f$ is analytic in $z$ for $|z|<\gamma, f \in \mathfrak{D}$. The purpose of this note is to investigate the set $Z$ of points $z$ where $T(z)$ is normal. ${ }^{2}$ The set $Z$, apart from degenerate cases, is shown in Theorem 6 to consist of one or more analytic arcs through the point $z=0$. The nature of $Z$ is of interest in the analytic perturbation of normal operators. An application of Theorem 6 to this theory is given in Theorem 7 .

2. Preliminary facts. It is convenient to state some known facts upon which our results are based. The real zeros of an analytic function $\phi(\xi, \eta)$ are studied in the following theorem of Bliss:

THEOREM 1. Let $\phi$ be a complex valued function of the two complex variables $\xi$ and $\eta$ which is defined by a convergent series $\phi(\xi, \eta)$ $=\sum_{m, n=0}^{\infty} a_{m n} \xi^{m} \eta^{n}$ about the origin. Let $\phi(0,0)=0$. Then there exists $a$ neighborhood $N$ of $(0,0)$ such that the set $Z$ of those real pairs $(x, y)$ which lie in $N$ and satisfy $\phi(x, y)=0$ consists of a finite number of distinct arcs. Each such arc has a representation of the form

$$
x=a t^{p}, \quad y=b t^{\mu}+b^{\prime} t^{\prime \prime}+\cdots, \quad 0 \leqq t<t_{1},
$$

where the exponents are integers, the coefficients are real, and $a$ and $b$ are not both zero.

We use the following two theorems of Rellich about symmetric operators with fixed domain:

THEOREM 2. Let $T(x)$ be a family of symmetric operators depending on the real parameter $x$, having a fixed domain $\mathfrak{D}$, for $x$ in a neighborhood of $x=0$. Let $T(x) f$ be analytic in $x$ for $f \in \mathfrak{D}$, and suppose $T(0)$ is self-adjoint. Then $T(x)$ is self-adjoint for $x$ in a neighborhood of $x=0$, [4, III, p. 561].

Presented to the Society, April 20, 1957 under the title $A$ proof of Rellich's theorem for normal operators; received by the editors July 21, 1955 and, in revised form, September 14, 1956.

${ }^{1}$ Work performed at the University of California under Professor F. Wolf for the Office of Ordnance Research, U. S. Army, Contract No. DA-04-200-ORD-171.

2 For basic definitions see $[4 ; 6 ; 8]$. A definition of unbounded normal operator is given in [6]. 
THEOREM 3. If $T(x)$ satisfies the hypothesis of Theorem 2 and if in addition for some $\mu$ in the resolvent set of $T(0)$ the operator $(\mu-T(0))^{-1}$ is completely continuous then there exists $\epsilon>0$ and a set of functions $\left\{\nu_{i}(x)\right\}$ analytic for $-\epsilon<x<\epsilon$, such that for $-\epsilon<x<\epsilon$ the spectrum of $T(x)$ consists of the set of points $\left\{\nu_{i}(x)\right\}[4, \mathrm{~V}, \mathrm{p} .478]$.

We shall need the following lemma about self-adjoint operators depending on two real parameters:

Lemma 1. Let $S(x, y)$ be a family of self-adjoint operators depending on the real parameters $x, y$, and having a fixed domain $\mathfrak{D}$, for $x, y$ in $a$ neighborhood of $(0,0)$. Let a set of linear operators $S_{m n}, m, n=1,2, \cdots$ exist with domain $\mathfrak{D}$ such that for $f \in \mathcal{D}$

$$
S(x, y) f=\sum_{m, n=0}^{\infty} S_{m n} f x^{m} y^{n}
$$

and the series converges absolutely and uniformly in $x, y$ in a neighborhood of $(0,0)$. Then in some neighborhood of $(0,0)$ the Cayley transform $U(x, y)$ of $S(x, y)$ is a bounded operator which is expressible as an absolutely and uniformly convergent series in $x, y$ whose coefficients are bounded operators.

Proof. Let $R=(-i-S(0,0))^{-1}$. Since $-i$ is in the resolvent set of $S(0,0)$ the operator $R$ is bounded with range $\mathfrak{D}$. Let $\gamma>0$ be such that (2.2) is absolutely and uniformly convergent in $x, y$ for $|x|+|y| \leqq \gamma$. If $\xi, \eta$ are complex numbers the series $\sum_{m, n-0}^{\infty} S_{m n} f \xi^{m} \eta^{n}$ is absolutely and uniformly convergent in $\xi, \eta$ for $|\xi|+|\eta| \leqq \gamma$ and defines an operator $S(\xi, \eta)$ dependent on $\xi, \eta$. The operator $S(\xi, \eta) R$ is bounded since it is closed and has domain $\mathfrak{S}$. Let $M=\max _{|\xi|+|\eta| \leq \gamma}\|S(\xi, \eta) R\|$. From the identity

$$
S_{m n} R=-\frac{1}{4 \pi} \int_{|\eta|=\gamma} \int_{|\xi|=\gamma} S(\xi, \eta) R \xi^{-m-1} \eta^{-n-1} d \xi d \eta
$$

it follows by taking norms that

$$
\left\|S_{m n} R\right\|<M \gamma^{-m-n} .
$$

By definition the Cayley transform is

$$
\begin{aligned}
U(x, y) & =(i-S(x, y))(-i-S(x, y))^{-1} \\
& =\left(i-S(x, y) R R^{-1}(-i-S(x, y))^{-1} .\right.
\end{aligned}
$$

Using (2.4) it follows that the operator $(+i-S(x, y)) R$ is expressible as an absolutely and uniformly convergent power series in $x, y$ for 
$|x|+|y| \leqq \gamma / 2$, whose coefficients are bounded operators. Also using (2.4) for $|x|+|y| \leqq \gamma(1+M)^{-1 / 2}$

$$
\|(S(x, y)-S(0,0)) R\| \leqq \sum_{m+n=1}^{\infty}\left\|S_{m n} R\right\||x|^{m}|y|^{n}<1
$$

and the series

$$
-R \sum_{\nu=0}^{\infty}\left(-\sum_{m+n=1}^{\infty} S_{m n} R x^{m} y^{n}\right)^{\nu}
$$

is absolutely and uniformly convergent in $x, y$. The series (2.6) may be rearranged as a power series in $x, y$ since it converges absolutely and uniformly. It is known (cf. $[8$, p. 326]) that

$$
\begin{aligned}
(-i-S(x, y))^{-1} & =-R(1+(S(x, y)-S(0,0)) R)^{-1} \\
& =-R \sum_{\nu=0}^{\infty}\left(-\sum_{m+n=1}^{\infty} S_{m n} R x^{m} y^{n}\right)^{\nu} .
\end{aligned}
$$

Therefore the operator $(-i-S(x, y))^{-1}$ may be written as an abso lutely and uniformly convergent series in $x, y$ for $|x|+|y|$ $\leqq \gamma(1+M)^{-1} / 2$. Since by $(2.5) U(x, y)$ is the product of two operators, $\left(i-S(x, y) R\right.$ and $R^{-1}(-i-S(x, y))^{-1}$, which are expressible as absolutely and uniformly convergent series in a neighborhood of $(0,0)$ it must have the same property.

The connection between the spectrum of a normal operator and that of corresponding self-adjoint operators is given by the following theorem of Jamison. Although Jamison has stated this theorem for bounded normal operators the proof extends step for step to the unbounded case.

THEOREM 4. Let $T$ be a normal operator and let $\mu$ belong to the spectrum of $T$. If $S_{1}, S_{2}$ are defined on the domain of $T$ by the equations

$$
S_{1}=\left(T+T^{*}\right) / 2, \quad S_{2}=\frac{1}{2 i}\left(T-T^{*}\right)
$$

then $\operatorname{Re} \mu$ is in the spectrum of $S_{1}$ and $\operatorname{Im} \mu$ is in the spectrum of $S_{2}$, [3, p. 104].

3. The set $Z$. Before investigating the zero set $Z$ of an analytic operator, which is done in Theorem 6 , it is convenient to consider the zero set of an analytic element of an arbitrary Banach space $\ell^{3}$ This is done in Theorem 5 which is based on the following lemma:

\footnotetext{
${ }^{3}$ I am indebted to the referee for this preliminary formulation of the problem.
} 
LeMma 2. Let $f$ be a function of the two complex variables $\xi$ and $\eta$ with values in a Banach space $\mathbb{R}$ which is defined in a neighborhood of the origin by a convergent series $f(\xi, \eta)=\sum_{m, n=0}^{\infty} f_{m n} \xi^{m} \eta^{n}, f_{m n} \in \mathbb{R}$. Let $f(0,0)=0$. Suppose that $f(\xi, \eta) \not \equiv 0$, and that in every neighborhood of $(0,0)$ there is a nonzero real pair $(x, y)$ such that $f(x, y)=0$. Then there exists a neighborhood $N_{1}$ of $(0,0)$ such that the set $Z$ of those real pairs $(x, y)$ lying in $N_{1}$ and satisfying $f(x, y)=0$ consists of a finite number of arcs each of which has a representation of the form (2.1).

Proof. Choose $\gamma>0$ such that the series for $f(\xi, \eta)$ converges for $|\xi|+|\eta|<\gamma$. For $f^{*} \in \Omega^{*}$ we have $f^{*}(f(\xi, \eta))=\sum_{m, n=0}^{\infty} f^{*}\left(f_{m n}\right) \xi^{m} \eta^{n}$ so that the functions $f^{*}(f(\xi, \eta))$ are expressible as convergent series in $\xi, \eta,|\xi|+|\eta|<\gamma$. Since by assumption $f(0,0)=0$ it follows for all $f^{*} \in \Omega^{*}, f^{*}(f(0,0))=0$. Also the assumption $f(\xi, \eta) \not \equiv 0$ implies there must exist at least one $f_{1}^{*}$ such that $f_{1}^{*}(f) \not \equiv 0$.

The function $f_{1}^{*}(f)$ satisfies the hypothesis of Theorem 1. Therefore by Theorem 1 we may choose a neighborhood $N$ of $(0,0)$ and $t_{1}$ such that the real zero's of $f_{1}^{*}(f(\xi, \eta))=0$ consist of a finite number of arcs of the form (2.1).

Let $C=\{x(t), y(t)\}$ be an arc of the form (2.1) on which $f_{1}^{*}(f)=0$. The arc $C=\{x(t), y(t)\}$ will be defined to be a common arc for $f$ if for each functional $f^{*}$ and each interval $0<t<t_{0}$ there exists a $t^{*}$, $0<t^{*}<t_{0}$, such that $f^{*}\left(f\left(x\left(t^{*}\right), y\left(t^{*}\right)\right)\right)=0$. If an arc $C$ is a common arc then we have $f^{*} f(x(t), y(t)) \equiv 0$ for all $f^{*} \in \Omega^{*}$ since $f^{*} f(x(t), y(t))$ is analytic in $t, 0<t<t_{1}$, and hence $f(x(t), y(t)) \equiv 0$. If $C$ is not a common arc then for some $f^{*}$ we must have $f^{*} f(x(t), y(t)) \neq 0$ in some interval $0<t<t_{0}$ and therefore $f(x(t), y(t)) \neq 0$ in that interval. Choose a neighborhood $N_{1}$ of $(0,0)$ such that for all of the arcs which are not common $\operatorname{arcs} f(x(t), y(t)) \neq 0$ for $x(t), y(t)$ in $N_{1}, t \neq 0$. Then $f$ vanishes in $N_{1}$ only on common arcs and $f$ vanishes identically on these, which was to be shown.

Using Lemma 2 we have the following theorem:

TheOREM 5. If $f(x, y)$ is a function of two real variables $x, y$ into a Banach space $\mathbb{R}$, whose values for $|x|+|y| \leqq \gamma$ can be given by an absolutely and uniformly convergent series in $x, y$ which vanishes at the origin, then in some neighborhood of the origin the zero set $Z$ of this function either (i) reduces to a point, or (ii) fills a full neighborhood of the origin, or (iii) consists of a finite number of analytic arcs.

PROOF. Let $\sum_{m^{*} n=0}^{\infty} f_{m n} x^{m} y^{n}, f_{m n} \in \mathcal{R}$ be the series for $f(x, y)$. If $\xi, \eta$ are complex numbers then the series $\sum_{m \cdot n=0}^{\infty} f_{m n} \xi^{m} \eta^{n}$ converges for $|\xi|+|\eta|<\gamma$ and defines a function $f(\xi, \eta)$ which coincides with $f(x, y)$ when $\xi, \eta_{-}$are real. 
If $f(x, y) \equiv 0$ then conclusion (ii) holds. If $f(x, y) \not \equiv 0$ then either every neighborhood of the origin $(0,0)$ contains a point $x, y$ such that $f(x, y)=0$ or else there exists some neighborhood of the origin in which $f(x, y)$ does not vanish, except for $x=y=0$. In the latter case conclusion (i) holds and in the former case conclusion (iii) holds since we may apply Lemma 2 to $f(\xi, \eta)$.

We now apply the previous theorem to an analytic operator.

Theorem 6. Let $T(z)$ be a closed operator on $\mathfrak{S}$ depending on a complex parameter $z,|z|<\gamma$, satisfying the properties:

(a) $T(z), T^{*}(z)$ have fixed domains $\mathfrak{D}$ for $|z|<\gamma$.

(b) $T(z) f, T^{*}(\bar{z}) f$ are analytic in $z,|z|<\gamma, f \in \mathfrak{D}$.

(c) $T(0)$ is a normal operator.

Then in some neighborhood of the origin the set $Z$ of points $z$ where $T(z)$ is normal either (i) reduces to a single point or (ii) fills a full neighborhood of the origin or (iii) consists of a finite number of analytic arcs.

Proof. Define a set of operators $T_{k}, k=1,2, \cdots$ on $\mathfrak{D}$ by letting $T_{k} f=g_{k}$ where $g_{k}$ is the coefficient of the $k$ th term of the series for $T(z) f$. The operators so defined are linear. A result of Sz.-Nagy [5, Theorem 4] states that if $T(z)$ is a closed operator with fixed domain $\mathfrak{D}$ for $|z|<\gamma$ and $T(z) f=\sum_{\boldsymbol{k}=0}^{\infty} T_{k} f z^{k}$ for $f \in \mathfrak{D},|z|<\gamma$, where $T_{k}$ are linear with domain $\mathfrak{D}$, then constants $a \geqq 0, b \geqq 0, p \geqq 0$ exist such that

$$
\left\|T_{k} f\right\| \leqq p^{k-1}(a\|f\|+b\|T(0) f\|), \quad k=1,2, \cdots .
$$

From (3.1) it follows that $T(z) f$ can be written as an absolutely and uniformly convergent series in $x, y, x=\operatorname{Re} z, y=\operatorname{Im} z,|x|+|y|$ $<p^{-1} / 2$. Similarly $T^{*}(z) f$ can be written as an absolutely and uniformly convergent series in $x, y$ in some neighborhood of $(0,0)$.

Define operators $S_{1}(z), S_{2}(z)$ corresponding to $T(z)$ on $\mathfrak{D}$ by (2.7). The operators $S_{1}(0), S_{2}(0)$ are commuting self-adjoint operators since $T(0)$ is normal. By (2.7) the elements $S_{1}(z) f, S_{2}(z) f$ may be expanded in absolutely and uniformly convergent series in $x, y$ of the form (2.3) in a neighborhood of $(0,0)$ since $T(z) f, T^{*}(z) f$ can be. Also by (2.7) $S_{1}(z), S_{2}(z)$ are symmetric for $|z|<\gamma$.

Using Theorem 2, $S_{1}(x), S_{2}(x)$ are self-adjoint for real $x$ in a real neighborhood of $x=0,-\epsilon_{1}<x<\epsilon_{1}$. Next for fixed $x,-\epsilon_{1}<x<\epsilon_{1}$, we apply Theorem 2 to $S_{1}(z), S_{2}(z)$ as functions of $y$ to conclude that $S_{1}(z), S_{2}(z)$ are self-adjoint for $y$ in a neighborhood of the $x$-axis, $-\epsilon<y<\epsilon$. By reference to the proof of Theorem 2 [4, III, p. 561] it may be shown by a straightforward argument that $\epsilon$ may be chosen independent of $x,-\epsilon_{1}<x<\epsilon_{1}$ so that $S_{1}(z), S_{2}(z)$ are self-adjoint in a complex neighborhood of $z=0$. 
Let $U_{1}(z), U_{2}(z)$ be the Cayley transforms of the self-adjoint operators $S_{1}(z), S_{2}(z)$. The operators $U_{1}(z), U_{2}(z)$ are elements of the Banach space $\&$ of bounded operators. The operators $U_{1}(0), U_{2}(0)$ commute since $S_{1}(0), S_{2}(0)$ commute. By Lemma 1 the operators $U_{1}(z), U_{2}(z)$ may be written as absolutely and uniformly convergent series in $x, y$ in a neighborhood of $(0,0)$. Let $f(x, y)=U_{1}(z) U_{2}(z)$ $-U_{2}(z) U_{1}(z)$. The operator $f(x, y)$ may be written as an absolutely and uniformly convergent series in $x, y$ in a neighborhood of $(0,0)$. Also $f(0,0)=U_{1}(0) U_{2}(0)-U_{2}(0) U_{1}(0)=0$. The operator $f(x, y)$ therefore satisfies the hypothesis of Theorem 5 so that the zero set $Z$ of $f(x, y)$ has the form stated in the conclusion of Theorem 5 . Since $Z$ is the set where $U_{1}(z), U_{2}(z)$ commute it follows that $Z$ is the set where the spectral measures of $U_{1}(z), U_{2}(z)$ commute. This implies that $Z$ is the set where $S_{1}(z), S_{2}(z)$ commute. A necessary and sufficient condition that $T(z)$ be normal is that $S_{1}(z), S_{2}(z)$ commute $[6$, p. 360] so that $T(z)$ must be normal for $z$ in the set $Z$. This proves the theorem.

4. Remarks. If $Z$ is a full neighborhood of $z=0$ and $T(z)$ is a bounded operator, $T(z)=\sum_{n=0}^{\infty} T_{n} z^{n}$, then S. L. Jamison has shown that $\left\{T_{n}\right\}$ is a commuting family of operators [2]. If $\operatorname{dim} \mathfrak{S}=m<\infty$ and $T(z)=A+z B$ where $A, B$ are normal operators $H$. Wielandt has shown that $Z$ is either a straight line, or a point, or a full neighborhood of $z=0$ [7]. He gives a sufficient condition for $Z$ to be a full neighborhood of $z=0$, in which case $A$ and $B$ commute.

5. An application. We apply Theorem 6 to prove a theorem about analytic perturbation of a normal operator whose inverse is a completely continuous operator. The proof is based on Theorem 3, which is the corresponding theorem for symmetric operators, together with Theorem 4.

TheOREM 7. Let $T(z)$ be a closed operator on $\mathfrak{S}$ depending on the parameter $z,|z|<\gamma$, and satisfying the properties:

(a) $T(z), T^{*}(z)$ have fixed domain $\mathfrak{D}$, for $|z|<\gamma$.

(b) $T(z) f, T^{*}(\bar{z}) f$ are analytic in $z,|z|<\gamma, f \in \mathfrak{D}$.

(c) $T(0)$ is a normal operator with a completely continuous inverse.

(d) $T\left(x_{n}\right)$ is normal on a set of real positive numbers $\left\{x_{n}\right\}$ such that $\lim _{n \rightarrow \infty} x_{n}=0$.

Then there exists $\delta>0$ and a set of functions $\left\{\mu_{i}(x)\right\}$, analytic for $0 \leqq x<\delta$, such that for $0 \leqq x<\delta$ the spectrum of $T(x)$ is equal to the set $\left\{\mu_{i}(x)\right\}$.

Proof. By Theorem 6 and hypothesis (d) there exists $\delta^{\prime}>0$ such that, for $0 \leqq x<\delta^{\prime}, T(x)$ is normal with domain $\mathfrak{D}$. 
Define the operators $S_{1}(x), S_{2}(x)$ for $-\delta^{\prime}<x<\delta^{\prime}$ by (2.7). These operators are symmetric for $-\delta^{\prime}<x<\delta^{\prime} . S_{1}(x) f, S_{2}(x) f$ are analytic in $x,-\delta^{\prime}<x<\delta^{\prime}, f \in \mathfrak{D}$, by hypothesis (b). Since by hypothesis (c) $T^{-1}(0)$ is completely continuous, $T(0)$ has a countable discrete spectrum. Choose a point $\alpha$ in the resolvent set of $T(0)$ such that $\operatorname{Im} \alpha \neq 0$. Since $S_{1}(0)$ is self-adjoint $\alpha$ is in the resolvent set of $S_{1}(0) .(\alpha-T(0))^{-1}$ is a completely continuous operator and $\left(\alpha-S_{1}(0)\right)^{-1}$ is a bounded operator. The second resolvent equation holds for $\alpha$ and is the easily verified identity:

$$
\begin{aligned}
& \left(\alpha-S_{1}(0)\right)^{-1} \\
& =(\alpha-T(0))^{-1}-\frac{1}{2}(\alpha-T(0))^{-1}\left(T(0)-T^{*}(0)\right)\left(\alpha-S_{1}(0)\right)^{-1}
\end{aligned}
$$

The operator $\left(T(0)-T^{*}(0)\right)\left(\alpha-S_{1}(0)\right)^{-1}$ is closed and defined for all $f \in \mathfrak{S}$ which implies that it is a bounded operator. By $(5.1)\left(\alpha-S_{1}(0)\right)^{-1}$ is the product of $(\alpha-T(0))^{-1}$ times a bounded operator so that it is completely continuous. A similar argument shows that, for some $\beta$, $\left(\beta-S_{2}(0)\right)^{-1}$ is completely continuous. We have now shown that $S_{1}(x), S_{2}(x)$ satisfy the hypothesis of Theorem 3 so that there exists $\delta>0$ and two sets of functions $\left\{\alpha_{i}(x)\right\}\left\{\beta_{i}(x)\right\}$ analytic in $x,-\delta<x$ $<\delta$, such that $\delta<\delta^{\prime}$ and for $-\delta<x<\delta$ the spectrum of $S_{1}(x)$ consists of $\left\{\alpha_{i}(x)\right\}$ and the spectrum of $S_{2}(x)$ consists of $\left\{\beta_{i}(x)\right\}$. Given any $\mu$ in the spectrum of $T(x), 0 \leqq x<\delta$, by Theorem 4 there is an element $\alpha_{i}(x)$ of the spectrum of $S_{1}(x)$ and an element $\beta_{j}(x)$ of the spectrum of $S_{2}(x)$ such that $\mu=\alpha_{i}(x)+i \beta_{j}(x)$. It is readily shown that if $\mu=\alpha_{i}\left(x_{0}\right)$ $+i \beta_{j}\left(x_{0}\right)$ is in the spectrum of $T\left(x_{0}\right)$ for a particular $x_{0}$ then $\mu(x)$ $=\alpha_{i}(x)+i \beta_{j}(x)$ is in the spectrum of $T(x)$ for all $x, 0 \leqq x<\delta$. Therefore for $0 \leqq x<\delta$ the spectrum of $T(x)$ consists of a set $\left\{\mu_{i}(x)\right\}$ of functions analytic in $x, 0 \leqq x<\delta$, as was to be shown.

Each individual eigenvalue $\mu_{i}(z)$ of Theorem 7 is known to be analytic in a complex neighborhood of $z=0$ (cf. $[3 ; 8]$ ). The conclusion of Theorem 7 cannot however be strengthened to assert that the set $\left\{\mu_{i}(z)\right\}$ are all analytic in a complex sphere $|z|<\delta$ since the eigenvalues $\mu_{i}(z)$ may have branches which approach the $x$-axis as $i \rightarrow \infty$. An example of this using symmetric operators is given in [4, $\mathrm{V}, \mathrm{p.483]}$.

\section{REFERENCES}

1. G. A. Bliss, Fundamental existence theorems, Amer. Math. Soc. Colloquium Publications vol. 3, 1913.

2. S. L. Jamison, Perturbation of normal operators, thesis, University of California, 1950. 
3. - Perturbation of normal operators, Proc. Amer. Math. Soc. vol. 5 (1954) pp. $103-110$.

4. F. Rellich, Störungstheorie der Spectralzerlegung, III, Math. Ann. vol. 116 (1939) pp. 555-570; V, Math. Ann. vol. 118 (1942) pp. 462-484.

5. B. v. Sz.-Nagy, Perturbations des transformations lineaires fermées, Acta Univ. Szeged. vol. 14 (1951) pp. 125-137.

6. F. Riesz and B. v. Sz.-Nagy, Leçons d'analyse fonctionnelle, Budapest, 1953.

7. H. Wielandt, Pairs of normal matrices with property L, Journal of Research of the National Bureau of Standards vol. 51 (1953) pp. 89-90.

8. F. Wolf, Analytic perturbation of operators in Banach spaces, Math. Ann. vol. 124 (1952) pp. 317-333.

University of California, Berkeley

UNIVERSITY OF WASHINGTON

\section{ON A THEOREM OF MAGNUS}

\section{EDWIN HEWITT AND EUGENE P. WIGNER ${ }^{1}$}

1. In a recent paper $[2],{ }^{2} \mathrm{~W}$. Magnus has shown that analogues of the Fourier inversion and Plancherel theorems hold for matrixvalued functions on the real line $R$. We propose to show that these theorems actually hold for an arbitrary locally compact Abelian group, and that Magnus's inversion integral (1. c. (1.4)) can be simplified. For all group- and integral-theoretic notation, terms, and facts used here without explanation, see [1].

2. Let $G$ be a locally compact Abelian group, written additively, with character group $X$. Elements of $G$ will be denoted " $s$ ", " $t$ ", and elements of $X$ by " $\chi$ ", with or without subscripts. The differential of Haar measure on $G[X]$ will be denoted $d t[d \chi]$ and these measures are to be so chosen that equality obtains in the Fourier inversion theorem [1, p. 143] and Plancherel's theorem [1, p. 145].

2.1. Let $U$ be a continuous $n$-dimensional unitary representation of $G$, so that: $U(s+t)=U(s) U(t)$ for all $s, t \in G ; U(0)=I$; and all coefficients $u_{j k}$ of $U$ are continuous functions on $G$. Then the reduction theorem states that there exist a unitary matrix $V$ and characters $\chi_{1}, \cdots, \chi_{n} \in X$ such that

Received by the editors February 16, 1956 and, in revised form, October 1, 1956.

1 The first-named author is a fellow of the John Simon Guggenheim Memorial Foundation.

2 Numbers in brackets refer to the bibliography. 